\title{
FLAVOR STRUCTURE OF THE NUCLEON AS REVEALED AT HERMES
}

\author{
H.E. JACKSON \\ Physics Division, Argonne National Laboratory \\ Argonne, Illinois 60439, USA \\ On behalf of the HERMES Collaboration
}

\begin{abstract}
The flavor structure of the nucleon as revealed in parton distributions (PDF's) is central to understanding the partonic structure of the nucleon. Recent data on unpolarized PDF's and their implications for the flavor- dependent quark helicity distributions are discussed. Results are presented for spin asymmetries in inclusive and semi-inclusive cross sections for production of pions, and kaons measured by the HERMES experiment in deep-inelastic scattering of polarized positrons on proton and deuterium Targets. A full 5 component extraction of polarized quark distributions for $u, d, \bar{u}, \bar{d}$, and $(s+\bar{s})$ is reported. Resulting valence quark distributions conform to results of earlier experiments. There is no evidence for a significant polarization of the light sea. In contrast to the conclusions inferred from studies of polarized inclusive scattering, a leading order analysis of the HERMES data suggests a zero or slightly positive polarization of the strange sea. There is no evidence for a measurable flavor asymmetry in the polarized distributions for the light sea.
\end{abstract}

\section{Introduction}

Parton distribution functions(PDF's) form the basis for the description of the flavor structure of the nucleon. The unpolarized parton distribution functions, $\mathrm{q}_{f}\left(\mathrm{x}, \mathrm{Q}^{2}\right)$, where $\mathrm{f}$ is the quark flavor, describe the mass structure of the nucleon. Polarized parton distribution functions, such as the longitudinal PDF, $\Delta \mathrm{q}_{f}\left(\mathrm{x}, \mathrm{Q}^{2}\right)$, provide information on the helicity distributions of the quarks, ie. the spin structure of the nucleon. Over the years a very detailed picture of unpolarized PDF's has emerged, but a number of questions remain, some with important implications for the spin structure 
of the nucleon. By contrast, for polarized PDF's, only broad features of parton helicity distributions are established. Detailed properties, such as the sea polarizations are poorly known. And there are no direct measurements of gluon polarization, or of contributions from parton orbital angular momenta. New experiments addressing these issues involving selective probes of various features of partonic structure, particularly spin structure, are in progress or about to be launched at HERMES, COMPASS, and RHIC. This paper will focus on recent progress in measurements of the flavor dependence of the helicity distributions of the nucleon as measured in the HERMES experiment. The discussion will begin with brief remarks on unpolarized PDF's and a discussion of models of PDF's and their implications for deep inelastic scattering(DIS) reactions, and in particular the flavor structure of polarized PDF's. This will be followed by a description of the HERMES experiment and the flavor tagging technique for isolating quark effects in parton distributions. Finally, new data on polarized quark helicity distributions and their implications will be discussed.

\section{Unpolarized parton distributions}

A new generation of parton distributions based on a global data analysis (CTEQ6) is now available [1]. The study is carried out mainly in the $\overline{M S}$ scheme with an improved error treatment, but in most respects the new PDF's do not differ substantially from the previous compilation, CTEQ5. Among the outstanding problems are lack of data on parton distributions at high $\mathrm{x}_{b j}$, uncertainties in the behaviour of the light sea flavor asymmetry for $\mathrm{x}_{b j}$ above 0.1 , and the fragmentary information on the distributions for strange quarks. From the HERMES point of view, the unresolved discrepency in $\mathrm{s}(\mathrm{x})$ as determined by dimuon $\nu$ data compared to results from charged and neutral current structure functions is particularly serious. The lack of knowledge of $\mathrm{s}(\mathrm{x})$ precludes a precise comparision of new results for $\Delta \mathrm{s}(\mathrm{x})$ with the unitary limit. The CTEQ compilation assumes $s=\bar{s}=0.2(\bar{u}+\bar{d})$. The decrepency between the CTEQ value for $\mathrm{s}(\mathrm{x})$ and other compilations is as much as $100 \%$.

The flavor asymmetry of the light sea has received much attention. The Gottfried sum rule which is given by the equation

$$
I_{G}=\int_{0}^{1}\left[F_{2}^{p}(x)-F_{2}^{n}(x)\right] \frac{d x}{x}=\frac{1}{3}-\frac{2}{3} \int_{0}^{1}\left[\bar{d}_{p}(x)-\bar{u}_{p}(x)\right] d x
$$

provides a measure of that asymmetry. The first evidence for an asymmetry came from the CERN experiment NA51, which reported the result, $I_{G}=0.235 \pm 0.026$, instead of the value $1 / 3$ expected for a symmetric sea. Subsequently, in a measurement of the Drell Yan process [2] the ratio, $\bar{d} / \bar{u}$ 


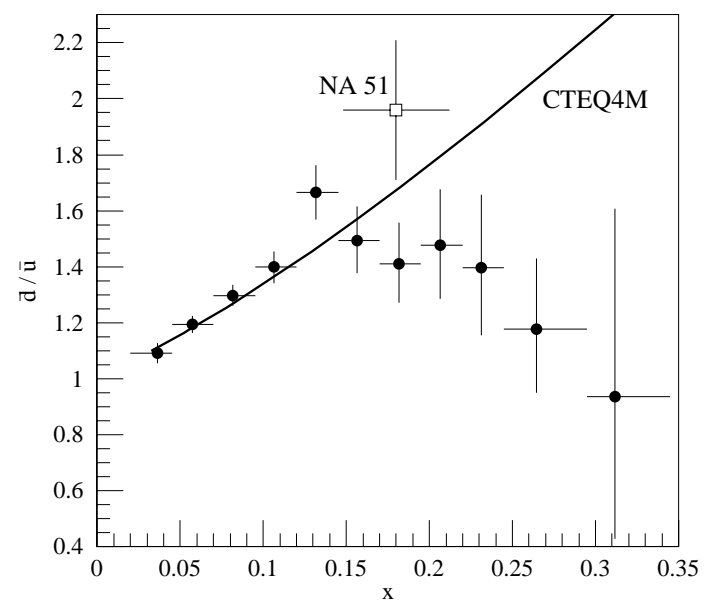

Figure 1. The ratio of $\bar{d} / \bar{u}$ as a function of the scaling variable, $\mathrm{x}$ measured in the Fermilab experiment E866. The result from NA51 is plotted as an open box. Also shown is the CTEQ4 prediction(solid line).

was measured as a function of $\mathrm{x}$. The results are presented in fig. (1). Important features of these data can be explained by a number of theoretical models, developed to describe PDF's. According to these models, the flavor aysmmetry observed for the light sea puts important constraints on the flavor asymmetry for the polarization of the light sea.

The chiral quark soliton model $(\chi \mathrm{QSM})$ makes a strong prediction [3] about $\Delta \bar{u}-\Delta \bar{d}$. In this approach the nucleon is described as a soliton of an effective field theory. Unpolarized quark and antiquark PDF's, as well as polarized PDF's for quarks plus antiquarks given by this model are in good agreement with experiment. From the structure of the theory, parametrically $\Delta \bar{u}(x)-\Delta \bar{d}(x)>|\bar{u}(x)-\bar{d}(x)|$. Calculations at low scale in the the large- $\mathrm{N}_{c}$ limit with an effective field theoretic description of chiral symmetry breaking confirm this prediction. The results are shown in Fig. (2). A second very different model employs methods of statistical mechanics. This statistical model in which one generates all the PDF's from a very small number of parameters [4] shows impressive agreement with the global data base for the structure functions $F_{2}^{p}\left(x, Q^{2}\right), F_{2}^{d}\left(x, Q^{2}\right)$, and, $G\left(x, Q^{2}\right)$. Again, from the chiral structure of QCD and features of $\mathrm{u}(\mathrm{x})$ and $\mathrm{d}(\mathrm{x})$ known from DIS, in this theory, $\bar{d}(x)>\bar{u}(x) ; \Delta \bar{u}(x)>$ $0 ; \quad \Delta \bar{d}(x)<0 ;$, and most significantly, $\Delta \bar{u}(x)-\Delta \bar{d}(x) \sim \bar{d}(x)-\bar{u}(x)$. A third model developed to explain the enhancement of $\bar{d}$ over $\bar{u}$ is a meson cloud model [5] in which the physical proton is viewed as a coherent sum of virtual meson-baryon states. The leading term is a proton with a symmetric 


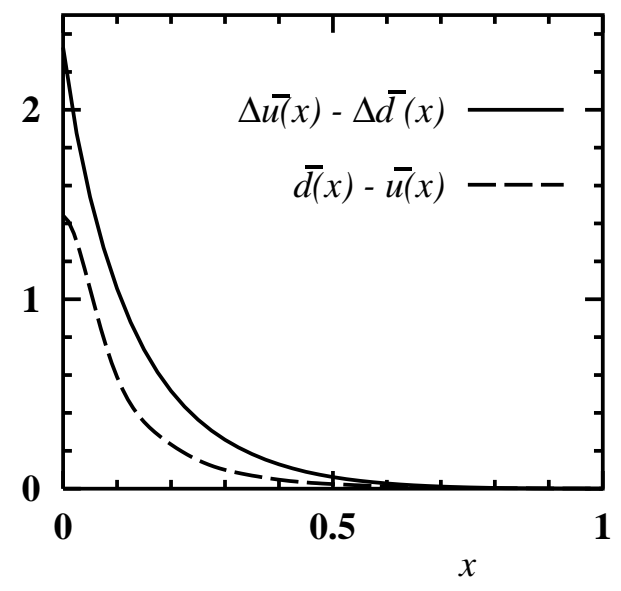

Figure 2. Isovector antiquark distributions at low normalization point, as obtained from the chiral quark soliton model.

sea. The "Sullivan process" generates an antiquark asymmetry through reactions which arise from the $\pi \mathrm{NN}$ and the $\pi \Delta \mathrm{N}$ couplings. With some tuning of the cutoff parameters for these couplings, the virtual pion model gives an excellent description of measured light sea asymmetry. However, in contrast to other models, for a light sea generated by a pion cloud plus a symmetric sea generated by gluon splitting, this model gives $\Delta \bar{u}-\Delta \bar{d} \approx 0$. Because of these differing predictions for the light sea polarization, a precise measurement of the asymmetry in the helicity distributions of the light sea will provide a good test of these models. A detailed measurement of the flavor structure of the quark helicity distributions is one of the principal goals of the HERMES experiment.

\section{The HERMES Experiment}

Deep inelastic scattering events are generated in the HERMES experiment by the interaction of the polarized lepton beam of the HERA accelerator at DESY with polarized target gases which are injected into a $40 \mathrm{~cm}$ long, tubular open-ended storage cell located at an interaction point on the lepton orbit. The lepton beam is self polarized by the Sokolov-Ternov effect with a polarization time which is typically about 20 minutes. The beam polarization is measured continuously with Compton backscattering of circularly polarized laser beams. The beam polarization is routinely about 0.55. Spin rotators in the ring provide longitudinal polarization at the interaction point. The polarized target gases, atomic $\mathrm{H}$ or $\mathrm{D}$ are generated by an atomic-beam source based on Stern-Gerlach separation which pro- 


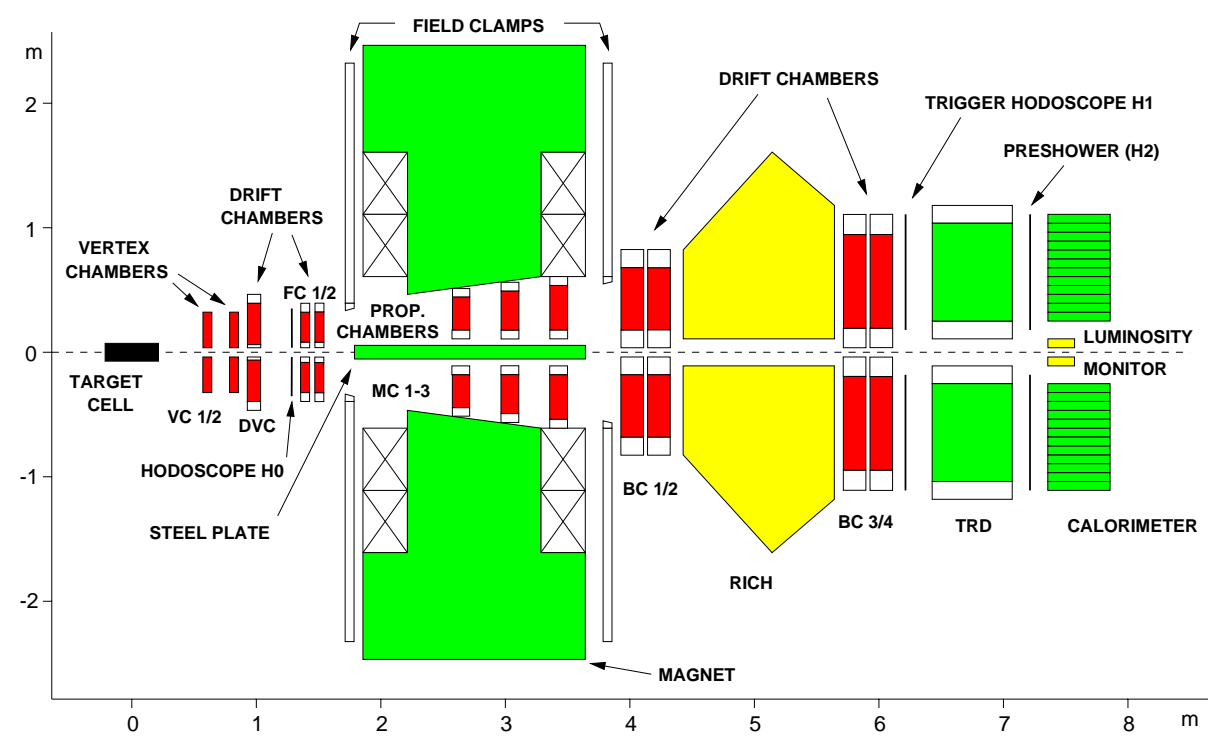

Figure 3. The HERMES Spectrometer.

vides an areal density of about $2 \times 10^{14}$ atoms $/ \mathrm{cm}^{2}$ for $\mathrm{H}$ and $7 \times 10^{13}$ atoms $/ \mathrm{cm}^{2}$ for $\mathrm{D}$. The nuclear polarization is measured with a Breit-Rabi polarimeter and the atomic fraction with a target gas analyser. The target polarization is reversed within short time intervals to minimize systematic effects. The relative lumimosity is measured by detecting Bhabha- or Mottscattered target electrons in coincidence with the scattered lepton, in a pair of $\mathrm{NaBi}\left(\mathrm{WO}_{4}\right)_{2}$ electromagnetic calorimeters.

The HERMES spectrometer[6], shown in Fig. (3) is a forward angle open geometry system consisting of two halves which are symmetric about a central horizontal shielding plate in the spectrometer magnet. A fly's eye calorimeter and a transition radiation detector furnish clean separation of hadrons and leptons. Identification of $\pi^{\prime} s, K^{\prime} s$ and $p^{\prime} s$ are accomplished by means of a novel dual-radiator ring-imaging Cerenkov counter(RICH)[7] which is located between the rear tracking chambers. The combination of radiators consisting of a wall of clear aerogel and a gas volume of $C_{4} F_{10}$ provide clean particle identification over almost the full acceptance of HERMES, i.e. 2-15 GeV. The scattered leptons and hadrons produced within an angular acceptance of $\pm 170 \mathrm{mr}$ horizontally, and 40 - $140 \mathrm{mrad}$ vertically are detected and identified. Typical kinematics for studies of DIS are $E=27.5 \mathrm{GeV}$ for the incident lepton, $x>0.02$ where $x=Q^{2} / 2 M \nu$ is the Bjorken scaling variable, $0.1 \mathrm{GeV}^{2}<Q^{2}<15 \mathrm{GeV}^{2}$ with $-Q^{2}$ the square of the momentum transfer, and $\nu<24 \mathrm{GeV}$ where $\nu=E-E^{\prime}$ with $E\left(E^{\prime}\right)$ 
is the energy of the incoming(scattered) lepton in the target rest frame. To insure that hadrons detected are in the current fragmentation region, cuts of $z=E_{h} / \nu>0.2$ and $x_{F} \approx 2 p_{\text {parallel }}^{h} / W>0.1$ are imposed, where $W=\sqrt{2 M \nu+M^{2}-Q^{2}}$ is the invariant mass of the photon-proton sytem.

\section{Flavor Decomposition}

Until recently, all of the information on the flavor decomposition of quark helicity distributions resulted from analysis of inclusive data under the assumption of $\mathrm{SU}(3)$ symmetry. One found [8]

$$
\Delta u=+0.78 \pm 0.03, \quad \Delta d=-0.48 \pm 0.03, \quad \Delta s=-0.14 \pm 0.03 .
$$

A subsequent next to leading order global analysis of all data for a proton target [9] gives for the strange quark contribution to the singlet axial coupling, $a_{s}=-0.07 \pm 0.04$. In order to improve sensitivity to flavor dependencies, the HERMES experiment uses semi-inclusive deep-inelastic scattering to determine the separate contributions $\Delta q_{f}(x)$ of the quarks and antiquarks of flavor $f$ to the total spin of the nucleon. By means of the technique of flavor tagging, individual spin contributions can be determined directly from spin asymmetries of hadrons with the appropriate flavor content. For example, the spin asymmetry of $K^{-}$, an all sea object, will have a high sensitivity to the polarization of the quark sea. The measured semi-inclusive spin asymmetry, $A_{\|}^{h}$, and the corresponding photon-nucleon asymmetry, $A_{1}^{h}$, for the hadron of type $h$ are given by

$$
A_{\|}^{(h)}=\frac{N_{(h)}^{\uparrow \downarrow}-N_{(h)}^{\uparrow \uparrow}}{N_{(h)}^{\uparrow \downarrow}+N_{(h)}^{\uparrow \uparrow}}, \quad A_{1}^{h}=\frac{A_{\|}^{h}}{D(1+\eta \gamma)},
$$

where, for simplicity, we assume unity beam and target polarizations and constant luminosity. Here $D$ is the depolarization factor for the virtual photon, $\eta$ is a kinematic factor, and $N^{\uparrow \uparrow}\left(N^{\uparrow \downarrow}\right)$ are the number of DIS events with coincident hadrons for target polarization parallel (anti-parallel) to the beam polarization. In leading order QCD assuming the validity of factorization, one can write the semi-inclusive DIS cross section, $\sigma^{h}\left(x, Q^{2}, z\right)$, to produce a hadron with energy fraction, $z=E_{h} / \nu$ as

$$
\sigma^{h}\left(x, Q^{2}, z\right) \propto \Sigma_{f} e_{f}^{2} q_{f}\left(x, Q^{2}, z\right) D_{f}^{h}\left(x, Q^{2}\right)
$$

where the sum is over quark and antiquark types $f=(u, \bar{u}, d, \bar{d}, s, \bar{s}) . E_{h}$ is the energy of the hadron. The quark charge, $e_{f}$, is in units of the elementary 

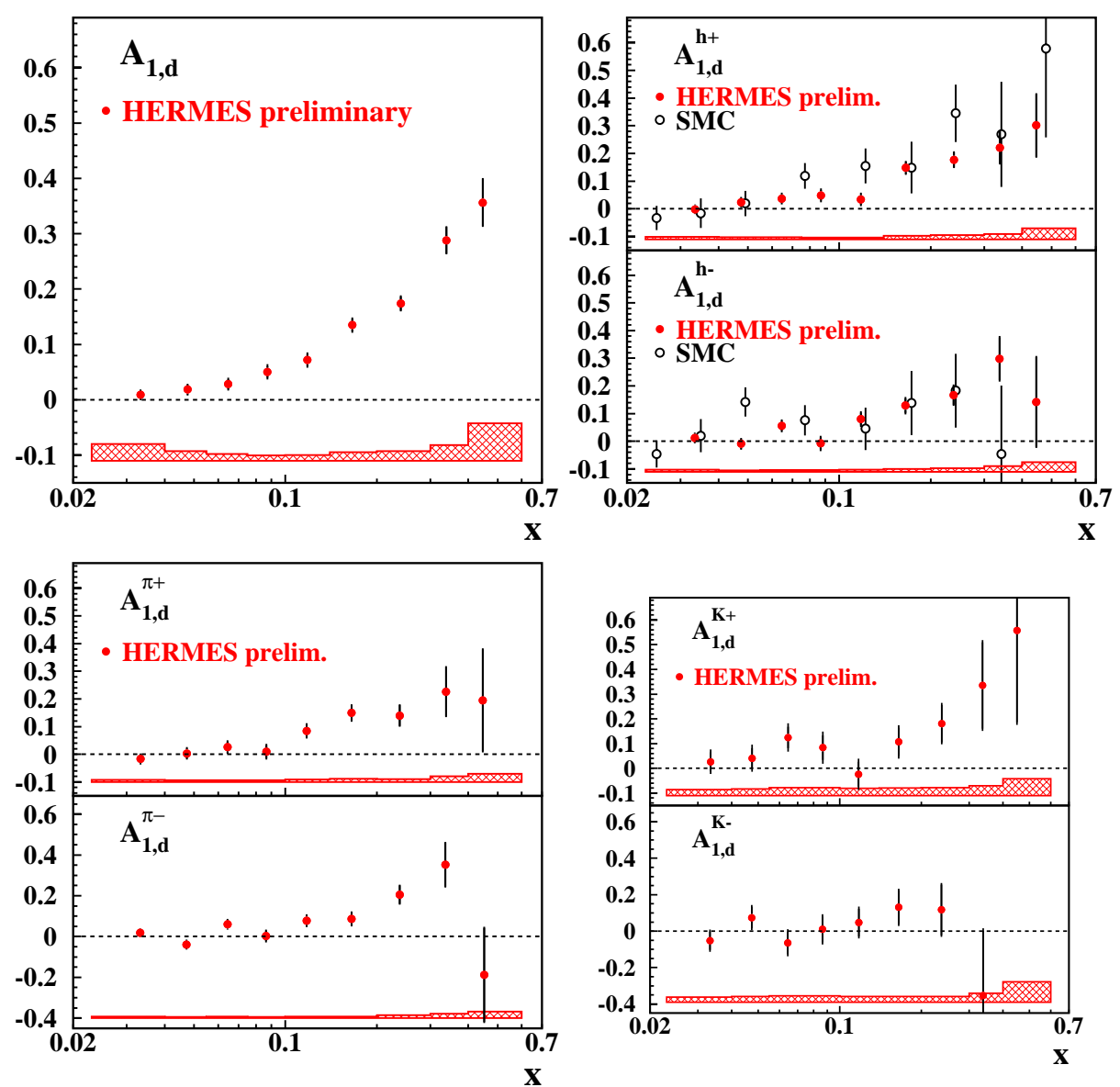

Figure 4. Inclusive and semi-inclusive hadron, pion and kaon asymmetries for a deuterium target. The hadron asymmetries are compared with data from the SMC collaboration. The error bars of the HERMES data are statistical and the bands are systematic uncertainties. These data form the deuteron portion of the data base used in the purity analysis described in the text.

charge. In this approximation,

$$
\begin{aligned}
A_{1}^{h}(x, z) & =\frac{\int_{z_{\min }}^{1} d z \sum_{f} e_{f}^{2} q_{f}(x) \cdot D_{f}^{h}(z)}{\int_{z_{\text {min }}}^{1} d z \sum_{f^{\prime}} e_{f^{\prime}}^{2} q_{f^{\prime}}(x) \cdot D_{f^{\prime}}^{h}(z)} \cdot \frac{\Delta q_{f}(x)}{q_{f}(x)} \cdot \frac{1+R\left(x, Q^{2}\right)}{1+\gamma^{2}} \\
& =\sum_{f} P_{f}^{h}(x) \frac{\Delta q_{f}(x)}{q_{f}(x)} \cdot \frac{1+R\left(x, Q^{2}\right)}{1+\gamma^{2}} .
\end{aligned}
$$

The quantities, $P_{f}^{h}(x)$, are the integrated purities which are defined by Eq. (6). They are spin-independent quantities in leading order and repre- 
sent the probability that the quark, $q_{f}$ was struck in the DIS event. The ratio $R=\sigma_{L} / \sigma_{T}$ of the longitudinal to transverse photon cross section corrects for the longitudinal component that is included in experimental parameterizations of $q_{f}\left(x, Q^{2}\right)$ but not in $\Delta q_{f}\left(x, Q^{2}\right)$. The term $\gamma=\sqrt{Q^{2}} / \nu$ is a kinematic factor.

A Monte Carlo method based on a DIS physics event generator is used to calculate the purities from CTEQ5 leading order parameterizations of the parton distributions. A LUND string fragmentation model tuned to HERMES kinematics provide fragmentation probabilities. The Monte Carlo program includes the effects of the acceptance of the experiment. The systematic uncertainties in the purities were estimated by variation of the fragmentation parameters and by using alternate PDF parameterizations. By incorporating the correction factor in the purities, one can rewrite Eq. (6) in a matrix form as

$$
\mathbf{A}(\mathbf{x})=\mathbf{P}(\mathbf{x}) \cdot \mathbf{Q}(\mathbf{x})
$$

where $\mathbf{A}(\mathbf{x})$ becomes a vector whose elements are all the integrated measured asymmetries which are to be included in the analysis. The $\mathbf{Q}(\mathbf{x})$ vector contains the quark and antiquark polarizations. These quantities are now connected by the purity matrix which contains the effective integrated purities. The determination of the quark polarizations from the experimentally measured spin asymmetries is reduced to the task[10] of inversion of Eq. (7) to obtain $\mathbf{Q}(\mathbf{x})$. Eq. (7) can be solved for $\mathbf{Q}(\mathbf{x})$ by minimizing

$$
\chi^{2}=(\mathbf{A}-\mathbf{P} \cdot \mathbf{Q})^{\mathbf{t}} V_{A}^{-1}(\mathbf{A}-\mathbf{P} \cdot \mathbf{Q})
$$

where $V_{A}$ is the covariance matrix of the asymmetry vector $\mathbf{A}(\mathbf{x})$. The flavor decomposition is obtained by solving Eq. (7) for a vector $\mathbf{Q}(\mathbf{x})$ of dimension corresponding to number of independent quark flavor distributions.

\section{Results}

The purity formalism has been used in the HERMES analysis to make a flavor decomposition into polarized quark distributions for $u, \bar{u}, d, \bar{d}$, and $s+\bar{s}$. For the first time, a global analysis of inclusive spin asymmetries and semi-inclusive spin asymmetries for $\pi^{+}, \pi^{-}, K^{+}$, and $K^{-}$has been carried out for longitudinally polarized targets of hydrogen, and deuterium. The measured spin asymmetries $A_{1}^{h}\left(x, Q^{2}, z\right)$ were integrated in each $x$ bin over the corresponding $Q^{2}$-range and the $z$-range from 0.2 to 1 to yield $A_{1}^{h}(x)$. The data for $A_{1}^{h}(x)$ together with the inclusive data obtained with the deuterium target are shown in Fig. (4). There the data for unidentified hadrons are compared to earlier results from the SMC collaboration [11] 


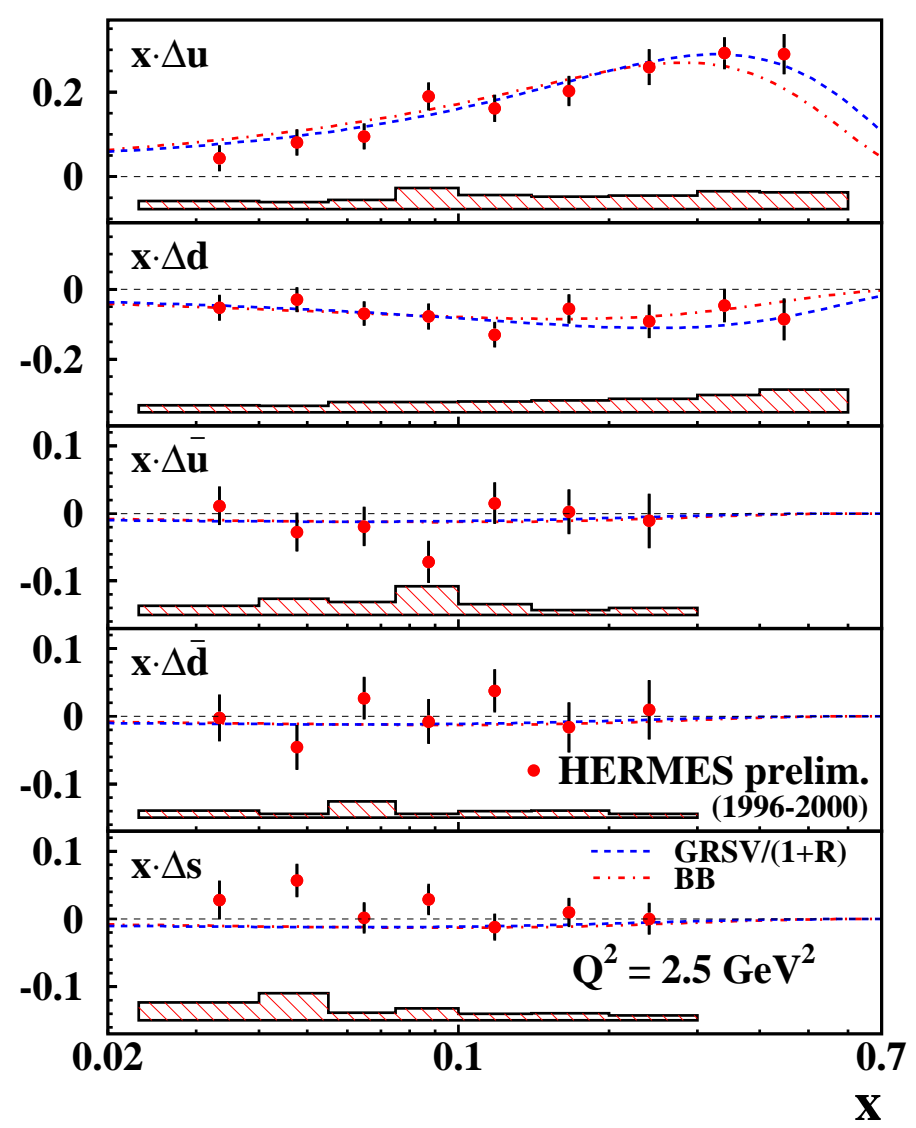

Figure 5. The $x$-weighted polarized quark densities. The plots show a five parameter fit to the data assuming a symmetric strange sea polarization. The data have been evolved to a common $Q^{2}=2.5 \mathrm{GeV}^{2}$. The dashed line shows a GRSV parameterization, and the dashed-dotted curve an alternate parameterization of Bluemlein and Boettcher (hep-ph/0203155).

The results of the decomposition obtained by solving Eq. (7) are presented in in Fig. (5). A symmetric strange sea polarization was assumed, i. e. $\Delta s / s=\Delta \bar{s} / \bar{s}$. The general features of quark densities follow those of earlier decompositions[10, 11]. The $u$-quarks show a strong positive polarization, while the $d$-quarks have a substantial negative polarization. The non-strange sea quarks are not significantly polarized. However, the strange sea appears to be positively polarized, contrary to the conclusions drawn within leading order QCD[12] from earlier inclusive data. The triplet 


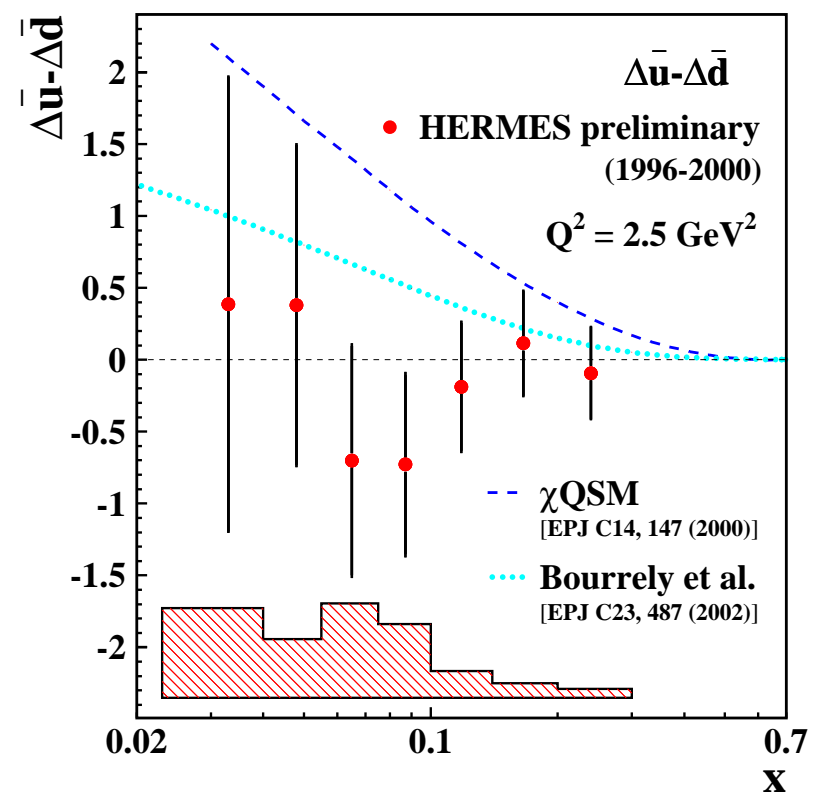

Figure 6. Flavor asymmetry $\Delta u-\Delta d$ of the light sea extracted from the HERMES five-component purity analysis. The curves describe predictions of the $\chi$ QSM model and the statistical model (see text). The error bars give statistical uncertainties and the shaded band the systematic error.

strength $\Delta q_{3}=\Delta u-\Delta d$ extracted from the HERMES data is in agreement with the Bjorken sum rule. The polarization of the strange sea can be extracted directly from the same data set by means of a purity analysis which uses only two spin asymmetries, $A_{1}^{D}(x)$ and $A_{1, D}^{K^{+}+K^{-}}(x)$. For this analysis, fragmentation functions from $e^{+} e^{-}$collider experiments can be used to calculate purities. This method measures the quantity $\Delta s+\Delta \bar{s}$ with no assumption about strange sea symmetry, and provides an independent check of the result from the five-component decomposition. The results obtained show the same trend of positive strange sea polarization.

\section{Remarks and conclusions}

The HERMES result is the first "direct" five component decomposition of quark helicity distributions using flavor tagging. The distributions for $u, d$, and the nonstrange sea show trends similar to those inferred from inclusive measurements, i.e. $\Delta u>>0, \Delta d<0$, and $\Delta \bar{u}=\Delta \bar{d} \approx 0$. The result $\Delta(s+\bar{s})>0$ is unexpected, and it poses a challenge to our understanding of the quark sea. To the extent that the sea arises from gluon splitting, one would expect from the result for the strange sea that $\Delta \bar{u} \approx \Delta \bar{d}>0$, i. e. 
that the light sea should show similar trends. The precision of the result reported here is limited, but its implications are of such significance, that further measurements of improved accuracy should receive high priority. However, one point is clear. The hypothesis of a large negative strange sea polarization as an explanation for the apparent violation of the Ellis-Jaffe sum rule in inclusive scattering is ruled out.

The HERMES flavor decomposition does not support recent conjectures of a strong breaking of the flavor symmetry of the light sea. The results for the quantity $\Delta u-\Delta d$ are shown in Fig. (6) together with predictions based on the chiral quark soliton model[3] $(\chi \mathrm{QSM})$ and the statistical model of Bourrely, et al [4]. Although the statistics are limited, the data indicate that any flavor asymmetry in the nonstrange sea is substantially smaller than the prediction of the $\chi \mathrm{QSM}$, and each of the measured points lies below the prediction of the statistical model. At the same time the HERMES result is consisent with the expectation based on the simple pion-cloud model discussed in section 2, that the polarized asymmetry should be small. These results demonstrate that details of the flavor decomposition of the helicity distributions can play an important part along with those of the unpolarized distributions in providing insight into the mechanism by which the quark sea of the nucleon is generated.

Other details of the sea distributions are under study. The singlet strength, $\Delta q_{3}=\Delta u-\Delta d$, agrees to within $1 \sigma$ with the Bjorken sum rule which states

$$
\Delta q_{3}=\int_{0}^{1} \Delta q^{N S}(x) d x=\left|\frac{g_{a}}{g_{v}}\right| \times C_{Q C D}=1.01 \pm 0.05
$$

A direct test of $\mathrm{SU}(3)$ symmetry assumed in inclusive analyses carried out to date, can be made with the data from the flavor decomposition, by determining the octet strength $\Delta q_{8}=\Delta u+\Delta d-2 \Delta s$. The octet combination can be related with $\mathrm{SU}(3)$ symmetry to the hyperon decay constants $F$ and $D$ according to the relation

$$
\Delta q_{8}=(3 F-D) \times C_{Q C D}=0.46 \pm 0.03
$$

where $C_{Q C D}$ is taken from ref. [13]. Analysis of the HERMES data is continuing. A determination of $\Delta q_{8}$ is of high priority. In summary, the HERMES results are the first complete decomposition of the quark helicity contributions to the spin of the nucleon. Such data will be of great value in revealing manner in which the nucleon spin is generated by its partonic structure.

\section{Acknowledgements}

The support of the DESY management and staff and the staffs of the

collaborating institutions is gratefully acknowledged. The author wishes to 
thank Roy J. Holt for a careful reading of the manuscript. The author also acknowledges the massive efforts of all the HERMES collaborators which have made the program a success. This work was supported in part by the U.S. Department of Energy under Contract No. W-31-109 -ENG-38.

\section{References}

1. Pumplin, J., et al. (2002) New Generation of Parton Distributions with Uncertainties from Global QCD Analysis, hep-ph/0201195, 1-43.

2. Hawker, E. A., et al. (1998) Measurement of the Light Antiquark Flavor Asymmetry in the Nucleon Sea, Phys. Rev. Lett., 80, 3715 -3718.

3. Dressler, B., et al. (2000) Flavor asymmetry of polarized antiquark distributions and semi-inclusive DIS, Eur. Phys. J., C14, 147-157.

4. Bourrely, C., et al. (2002) A statistical approach for polarized parton distributions, Eur. Phys. J. C23, 487-501.

5. Thomas, A. W. (1983), Phys. Lett. 126B, 97-100.

6. Ackerstaff, K., et al. (1998) The HERMES Spectrometer, Nucl. Inst. Meth. A417, 230-265.

7. Akapov, A., et al. (2002) The HERMES dual-radiator ring imaging Cherenkov detector, Nucl. Inst. Meth. A479, 511-530.

8. Filippone, B. W. and Ji, X. (2001), The spin structure of the nucleon, hepph/010224, 1-70.

9. Adams, D., et al. (1997), Spin structure of the proton from polarized inclusive deepinelastic muon-proton scattering, Phys. Rev. D56, 5330-5358.

10. Ackerstaff, K., et al. (1999) Flavor decomposition of the polarized quark distributions in the nucleon from inclusive and semi-inclusive deep-inelastic scattering, Phys. Lett. B464, 123-134.

11. Adeva, B., et al. (1998) Polarised quark distributions in the nucleon from semiinclusive asymmetries, Phys. Lett. B420, 180-190.

12. Adeva, B., et al. (1994) Combined analysis of world data on nucleon spin structure functions, Phys. Lett. B320, 400-406.

13. Close, F. E. and Roberts. R. G. (1993) Consistent analysis of the spin content of the nucleon, Phys. Lett. B316, 165-171. 